\title{
Integral Quadratic Separation applied to polytopic systems
}

\author{
D. Peaucelle \\ LAAS-CNRS ; Université de Toulouse, \\ 7 av. du colonel Roche, F-31077 Toulouse, FRANCE \\ peaucelle@laas.fr
}

February 20, 2009

\begin{abstract}
Slack variables technique for parameter-dependent robustness analysis of polytopic systems has emerged about ten years ago and has spread for many stability and performance problems. One of its main advantage is the simplicity of use but it has the drawback to generate LMI problems with large number of decision variables are large sized constraints. The paper is devoted to the study of ways to limit this numerical burden while controlling the conservatism. The study is lead in the context of Integral Quadratic Separation thus covering a very large class of robust performance analysis problems.
\end{abstract}

\section{Keywords}

LMI, Robustness, Uncertain systems.

\section{Introduction}

Many results in robust control theory during the past decade have used a technique that we may call the slack variables technique. First presented in [3] for discrete-time systems, it was used for analysis of polynomial systems in [24], generalized for pole location analysis in [17], then to many other performance criteria for example in [5]. The technique has its main application to robustness analysis of affine polytopic uncertain systems. It consists in the introduction of auxiliary "slack" variables that decouple the uncertain data from the true decision variables related to the performance problem (such as the Lyapunov matrix etc.). The decoupling makes possible the search of parameter-dependent decision variables with LMI tools.

The main drawback of that method is the induced numerical complexity. One source of it is due to the large number of decision variables generated when considering one of these for each vertex of the polytope. As the number of vertices is most often of order $2^{p}$ where $p$ is the number of uncertain parameters, the numerical complexity makes the problem rapidly intractable. One may therefore choose to test a problem of reduced numerical burden consisting of searching for a unique set of decision variables for all vertices. The results are then more conservative but may be efficient in practice. This strategy is related to the well known "quadratic stability" framework proposed in [2]. But, in "quadratic stability" no slack variable is needed. Therefore the relationship between these two techniques must be studied. One of the contributions in the following is to perform this comparison.

The comparison between "quadratic stability" framework and slack variables technique is done for a very general formulation of robust analysis problems: the Integral Quadratic Separation (IQS) result for descriptor systems. IQS is a formulation inspired by topological separation of [21], by its reformulation in terms of LMIs for LTI systems in [9], [11] and takes as well advantage of Integral Quadratic Constraints (IQC) theory of [13]. The quadratic separation technique was proved to extend to descriptor systems in [18] and more recently considering integral quadratic separators in [19] to include most of known performance criteria having LMI formulation. It has many possible extensions as attested for example in many chapters of [6]. Thus, the comparison of "quadratic stability" framework with the slack variables technique is done for a very general class of robust analysis problems. Cases when "quadratic stability" based results exist are exhibited. For these cases, it is demonstrated that finding a unique set of decision variables needs not to use slack variables. 
The second source of increased numerical complexity is the large size of the slack variables and of the LMI constraints. When applied basically, the technique generates a slack variable proportional to the size of the matrices involved in the robust performance analysis description. The LMI constraints are of the same size. The second contribution is to demonstrate that not only it is possible to apply the technique generating smaller slack variables and smaller LMI constraints, but also that this can be done without increasing conservatism. In a few words: the slack variables to be generated need only to correspond to the actually uncertain data. The result generalizes those of [7] where slack variables were proved to be of no use in case of time-delay systems without uncertainties.

Globally the paper is devoted to the description of slack variable technique applied in the context of IQS. Starting from a very general result suitable for a very general class of robust performance analysis problems for descriptor systems, it then shows strategies to reduce the numerical burden while controlling the conservatism augmentation. At each step the general result is illustrated on impulse-to-norm performance (known to be equivalent to $H_{2}$ norm of LTI systems, see [22]).

The paper is organized as follows. The next section is devoted to preliminaries about IQS. Then, section III exposes the main results. The general slack variables theorem is produced, followed by a theorem for reducing the slack variable losslessly according to parameter-independent data. The section closes with the comparison with "quadratic stability". A conclusion ends the paper and exposes prospective software implementation of the results in RoMulOC toolbox, see [15].

\section{Preliminaries}

\section{$2.1 \quad$ Notations}

$\mathrm{R}^{m \times p}$ and $\mathrm{C}^{m \times p}$ are the sets of $m$-by- $n$ real and complex matrices respectively. $A^{*}$ is the transpose conjugate of the matrix $A$ (and the transpose if $A$ is real). $A$ is said to be Hermitian (symmetric in case of real matrices) if $A=A^{*} . \mathbf{1}_{a}$ and $\mathbf{0}_{a, b}$ are respectively the $a \times a$ identity matrix and the $a \times b$ zero matrix. Most often the dimensions are omitted but can be deduced from the context. For Hermitian matrices, the inequality $A>(\geq) B$ means that $A-B$ is positive (semi) definite. To shorten some formulas, $A^{\mathcal{H}}$ stands for the Hermitian matrix $A+A^{*}$.

$A^{\perp}$ denotes a full rank matrix whose columns span the null-space of $A$, while $A^{\circ}$ is a full rank matrix whose columns span the same space as the columns of $A$. Define as well $A^{\circledast}=A^{* \circ}$ which is such that the columns of $\left[\begin{array}{ll}A^{\perp} & A^{\circledast}\end{array}\right]$ span $\mathrm{C}^{m}$ (assuming $A \in \mathrm{C}^{n \times m}$ ).

The inner product used in the following is defined as follows $<f \mid g>=\operatorname{Tr}\left(\int_{0}^{\infty} f^{*}(t) g(t) d t\right)$ (where $\operatorname{Tr}$ is the trace operator) and $\|f\|=\sqrt{\langle f \mid f\rangle}$ is the related norm. $L_{2}$ denotes the set of measurable functions $f:\left[0,+\infty\left[\rightarrow C^{m \times p}\right.\right.$ with bounded norm $(\|f\|<\infty)$. An Integral Quadratic Constraint (IQC) is a constraint that involves a function $f$ and a matrix $\Theta$, defined as follows: $<f \mid \Theta f>\leq \mathbf{0}$.

\subsection{Well-posedness}

Let the following interconnected loop

$$
\mathcal{E} z(t)=\mathcal{A} w(t) \quad, \quad w(t)=[\nabla z](t)
$$

where $\mathcal{E}$ and $\mathcal{A}$ are constant matrices, possibly not square and that may not be full rank; $\nabla$ is an operator, that may be non-causal and may be uncertain, it is defined as belonging to a closed set $\nabla \in \nabla$.

The interconnected loop (1) is said to be well-posed if for all operators $\nabla \in \nabla$ the internal vectors characterizing the loop are unique and bounded. As described in details in [16] and [19], it corresponds to the following definition.

Definition 1 The interconnected systems (1) is said to be well-posed if whatever bounded signals $(\bar{z}, \bar{w})$ perturbating the system equations

$$
\mathcal{E} z(t)=\mathcal{A}(t) w(t)+\bar{z}(t) \quad, \quad w(t)=[\nabla z](t)+\bar{w}(t)
$$

and whatever uncertain operator $\nabla \in \nabla$, the internal signals $(\mathcal{E} z, w)$ are unique and bounded in $L_{2}$ :

$$
\exists \bar{\gamma}>0:\left\|\begin{array}{c}
\mathcal{E} z \\
w
\end{array}\right\| \leq \bar{\gamma}\left\|\begin{array}{c}
\bar{z} \\
\bar{w}
\end{array}\right\| \quad \begin{gathered}
\forall\left(\begin{array}{c}
\bar{z} \\
\bar{w}
\end{array}\right) \in L_{2} . \\
\forall \nabla \in \nabla
\end{gathered} .
$$


To illustrate the definition consider

$$
\left[\begin{array}{llll}
E & \mathbf{0} & \mathbf{0} & \mathbf{0} \\
\mathbf{0} & E & \mathbf{0} & \mathbf{0} \\
\mathbf{0} & \mathbf{0} & \mathbf{1} & \mathbf{0} \\
\mathbf{0} & \mathbf{0} & \mathbf{0} & \mathbf{1}
\end{array}\right]\left(\begin{array}{c}
\varphi_{0} x \\
\dot{x} \\
\varphi_{0} g \\
g
\end{array}\right)=\left[\begin{array}{cc}
\mathbf{0} & B \\
A & \mathbf{0} \\
\mathbf{0} & D \\
C & \mathbf{0}
\end{array}\right]\left(\begin{array}{l}
x \\
v
\end{array}\right)
$$

(where $\varphi_{0}$ is an operator used to represent non zero initial conditions in $L_{2}$ ). Interconnect it with the integration operator

$$
x(t)=x(0)+\int_{0}^{t} \dot{x}(\tau) d \tau=\left[\mathcal{I}\left(\begin{array}{c}
\varphi_{0} x \\
\dot{x}
\end{array}\right)\right](t)
$$

and the following uncertain operator

$$
v=\nabla_{i 2 n}\left(\begin{array}{c}
\varphi_{0} g \\
g
\end{array}\right): \quad v=\alpha \varphi_{0} \mathbf{1}_{m}, \quad|\alpha| \leq \frac{1}{\gamma}\left\|\begin{array}{c}
\varphi_{0} g \\
g
\end{array}\right\| .
$$

As demonstrated in [19], well-posedness of this interconnection is equivalent to $\gamma$ impulse-to-norm performance of the descriptor dynamic system

$$
\left\{\begin{array}{l}
E \dot{x}=A x+B v \\
g=C x+D v
\end{array}\right.
$$

(in the sense that, assuming zero initial conditions, the sum of the norms of the outputs $g$ resulting from bounded impulses on the different rows of $v$, is strictly less than $\gamma)$. In case of usual LTI systems $(E=\mathbf{1})$ and if $D=\mathbf{0}$, this performance is known to coincide with $H_{2}$ norm, see [22]. This example of impulse-to-norm performance is used in the following of the paper to illustrate the results.

Theorem 1 Let any factorization $\mathcal{E}=\mathcal{E}_{1} \mathcal{E}_{2}$ where $\mathcal{E}_{1}$ is a square full rank matrix. The interconnected loop (1) is well posed if there exists a Hermitian matrix $\Theta$ with bounded entries satisfying simultaneously the LMIs:

and the IQC:

$$
\left[\begin{array}{ll}
\mathcal{E}_{1} & -\mathcal{A}
\end{array}\right]^{\perp *} \Theta\left[\begin{array}{ll}
\mathcal{E}_{1} & -\mathcal{A}
\end{array}\right]^{\perp}>\mathbf{0}
$$

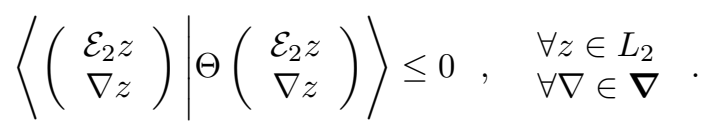

The proof of this result is a variation on the one provided in [9]. It moreover combines some of the properties used in quadratic separation demonstrations in [18] and [19]. Not to alleviate the paper the exact proof is not repeated here.

The matrix $\Theta$ defines a integral quadratic constraint which performs topological separation of graphs (see [21]) of operators involved in the loop (2). It is therefore called an integral quadratic separator (IQS) in the following.

\subsection{Building separators}

While condition (5) is an LMI and can thus be treated with efficient SDP tools, the second condition (6) is an IQC and needs some manipulations to be converted (at the expense of some conservatism) in LMI constraints on $\Theta$. This method is now described.

Assume the $\nabla$ operator is the sum of elementary operators as proposed in [23]

$$
\nabla=\sum_{j=1}^{\bar{\jmath}} J_{j} \nabla_{j} K_{j}
$$

where each element is independent of the others and belongs to a closed set $\nabla_{j} \in \nabla_{j}$ and where the $J_{j}$ matrices define an orthonormal basis: $J_{j_{1}}^{*} J_{j_{2}}=\mathbf{0}$ if $j_{1} \neq j_{2}$ and $J_{j}^{*} J_{j}=\mathbf{1}$. This modeling includes as a special case the block-diagonal structures of $\nabla$ operators. In such cases the matrices $J_{j}$ and $K_{j}$ are of the following form $J_{j}=\left[\begin{array}{lll}\mathbf{0} & \mathbf{1} & \mathbf{0}\end{array}\right]^{T}, K_{j}=\left[\begin{array}{lll}\mathbf{0} & \mathbf{1} & \mathbf{0}\end{array}\right]$ with appropriate dimentions. Define $L_{j}=K_{j}^{\perp}$, $G_{j}=\left(L_{j}^{*} \mathcal{E}_{2}^{*}\right)^{\perp}$ and $F_{j}=K_{j} \mathcal{E}_{2}^{*} G_{j}$. 
Theorem 2 If $\Theta_{1}, \ldots \Theta_{\overline{\mathrm{J}}}$ are IQS respectively with respect to each independent operator $\nabla_{1}, \ldots, \nabla_{\overline{\mathrm{J}}}$, i.e.

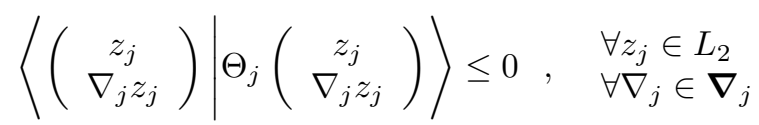

then, whatever $\Psi_{1}, \ldots \Psi_{\bar{\jmath}}$ constrained by the LMIs

$$
\left[\begin{array}{cc}
F_{j} F_{j}^{\circledast} & \mathbf{0} \\
\mathbf{0} & \mathbf{1}
\end{array}\right] \Psi_{j}\left[\begin{array}{cc}
F_{j} F_{j}^{\circledast} & \mathbf{0} \\
\mathbf{0} & \mathbf{1}
\end{array}\right]^{*} \leq \Theta_{j}
$$

and whatever $Y$, the following $\Theta$ (linearly dependent of the decision variables)

$$
\begin{aligned}
& \Theta=\left[\begin{array}{c}
\mathcal{E}_{2}{ }^{\perp *} \\
\mathbf{0}
\end{array}\right] Y+Y^{*}\left[\mathcal{E}_{2}^{\perp} \quad \mathbf{0}\right] \\
& +\sum_{j=1}^{\bar{j}}\left[\begin{array}{cc}
G_{j} F_{j}^{\circledast} & \mathbf{0} \\
\mathbf{0} & J_{j}
\end{array}\right] \Psi_{j}\left[\begin{array}{cc}
G_{j} F_{j}^{\circledast} & \mathbf{0} \\
\mathbf{0} & J_{j}
\end{array}\right]^{*}
\end{aligned}
$$

defines an IQS for condition (6).

The Theorem is a slight modification of that published in [16] (in fact the published version contains a mistake corrected in the version available at www.laas.fr/ peaucell/papers/cdc07a.pdf). The proof is therefore not reproduced here but the theorem is illustrated on the upper defined performance analysis problem.

An IQS with respect to the integration operator can be chosen as

$$
\Theta_{\mathcal{I}}=\left[\begin{array}{cc|c}
-P & \mathbf{0} & \mathbf{0} \\
\mathbf{0} & \mathbf{0} & -P \\
\hline \mathbf{0} & -P & \mathbf{0}
\end{array}\right]: P>\mathbf{0}
$$

while an IQS for the performance operator $\nabla_{i 2 n}$ is taking

$$
\Theta_{i 2 n}=\left[\begin{array}{cc|c}
-\tau \mathbf{1} & \mathbf{0} & \mathbf{0} \\
\mathbf{0} & -\tau \mathbf{1} & \mathbf{0} \\
\hline \mathbf{0} & \mathbf{0} & Q
\end{array}\right]: \operatorname{Tr}(Q)<\tau \gamma^{2} .
$$

Factorize $E=E_{1} E_{2}$ with $E_{1}$ square and full rank. The computations give

$$
\begin{gathered}
F_{1} F_{1}^{\circledast}=\left[\begin{array}{cc}
E_{2}^{*} E_{2}^{\circ} & \mathbf{0} \\
\mathbf{0} & E_{2}^{*} E_{2}^{\circ}
\end{array}\right], \quad F_{2} F_{2}^{\circledast}=\mathbf{1}, \\
G_{1} F_{1}^{\circledast}=\left[\begin{array}{cc}
E_{2}^{\circ} & \mathbf{0} \\
\mathbf{0} & E_{2}^{\circ} \\
\mathbf{0} & \mathbf{0} \\
\mathbf{0} & \mathbf{0}
\end{array}\right], G_{2} F_{2}^{\circledast}=\left[\begin{array}{cc}
\mathbf{0} & \mathbf{0} \\
\mathbf{0} & \mathbf{0} \\
\mathbf{1} & \mathbf{0} \\
\mathbf{0} & \mathbf{1}
\end{array}\right], \\
\mathcal{E}_{2}^{\perp}=\left[\begin{array}{c}
\operatorname{diag}\left(E_{2}^{\perp}, E_{2}^{\perp}\right) \\
\mathbf{0}
\end{array}\right] .
\end{gathered}
$$

LMIs may be easily coded. In the special case when $E_{2}=\mathbf{1}\left(E_{2}^{\circ}=\mathbf{1}\right.$ and $E_{2}^{\perp}$ is empty matrix) the formulas can be satisfied taking $\Psi_{\mathcal{I}}=\Theta_{\mathcal{I}}$ and $\Psi_{i 2 n}=\Theta_{i 2 n}$ and result in the following four LMIs:

$$
\begin{array}{cc}
A^{*} P+P A+\tau C^{*} C<\mathbf{0} & P>\mathbf{0} \\
Q<B^{*} P B+\tau D^{*} D & \operatorname{Tr}(Q)<\tau \gamma^{2}
\end{array}
$$

Many more types of uncertain operators $\nabla_{j}$ can be found in the literature related to other performances and/or robustness issues. Each elementary uncertain operator is associated to LMI constraints that describe (sometimes losslessly) sets of IQS candidates. See for example $D$-scallings, $D G$-scalling and vertex separators in [9]. See as well [10] for IQS candidates for frequency domain specifications. See [13] and [12] for separators in IQC theory. See also, in case of time-delay systems [25] and [8]...

Define by $\mathcal{L}_{j}\left(\Theta_{j}\right)$ the LMIs associated to an elementary uncertain operator set $\nabla_{j}$ such that if the LMIs are satisfied then $\Theta_{j}$ is an IQS that satisfies (7). Define as well the LMI constraints $\overline{\mathcal{L}}(\Theta)$ such that $\Theta$ satisfies constraints (9) with matrices $\Psi_{j}$ such that (8) and $\Theta_{j}$ matrices constrained by $\mathcal{L}_{j}\left(\Theta_{j}\right)$, i.e. $\Theta$ is an IQS with respect to $\boldsymbol{\nabla}$ if constrained by $\overline{\mathcal{L}}(\Theta)$. One then gets the following LMI result for well-posedness analysis. 
Theorem 3 If the LMI problem defined by $\overline{\mathcal{L}}(\Theta)$ and (5) is feasible, then the interconnected loop (1) is well-posed.

One can notice that almost all constraints $\mathcal{L}_{j}$ impose that the top-left block of the matrix $\Theta_{j}$ is negative semi-definite (this is a necessary condition when the zero operator $\nabla_{j}=\mathbf{0}$ belongs to the uncertainty set $\boldsymbol{\nabla}_{j}$ ). Because of that, it is in most cases not conservative to assume that the top-left block of $\Theta$ is semi-definite negative (i.e. $\left[\begin{array}{ll}\mathbf{1}_{a} & \mathbf{0}\end{array}\right] \Theta\left[\begin{array}{ll}\mathbf{1}_{a} & \mathbf{0}\end{array}\right]^{*} \leq \mathbf{0}$ where $a$ is the number of rows of $\mathcal{A}$ ).

\section{Main results}

In this study, $\mathcal{E}_{1}$ and $\mathcal{A}$ are assumed uncertain included in a polytope defined as

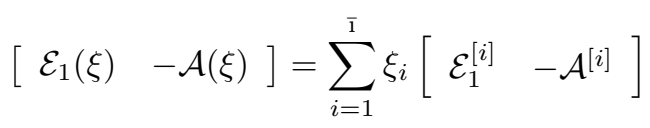

where $\xi=\left(\begin{array}{ccc}\xi_{1} & \ldots & \xi_{\overline{1}}\end{array}\right)$ is the uncertainty vector and lies in the unit simplex

$$
\xi \in \Xi=\left\{\xi_{i} \geq 0, \sum_{i=1}^{\overline{1}} \xi_{i}=1\right\} .
$$

This uncertain modeling corresponds to representations of parametric uncertainties. The $\xi$ vector represents the unknown deviations in the parameter values due to errors in identification or to inaccuracies of components issued from mass production. $\xi$ is assumed constant (or varying slowly compared to modeled dynamics).

Note that $\mathcal{E}_{1}$ being non singular, the descriptor characteristics of the system are all gathered in the $\mathcal{E}_{2}$ matrix. This $\mathcal{E}_{2}$ matrix is assumed certain (no uncertainties). Hence it is assumed that the uncertainties modify the parameters of the system but do not affect the intrinsic descriptor structure.

\section{1 "Slack varaibles" result}

Theorem 4 If there exists a unique matrix $H$ of appropriate dimensions and $\overline{1}$ IQS candidates $\Theta^{[i]}$ such that $\overline{\mathcal{L}}\left(\Theta^{[i]}\right)$ and

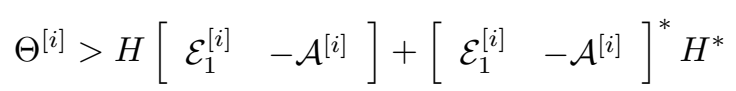

are feasible for all $i=1 \ldots \overline{1}$, then the interconnected loop (1) is well-posed whatever uncertainty $\xi \in \Xi$.

This result is nowadays classical and can be found in various versions in $[3,24,17,4,5]$ and many others. It has the advantage of its simplicity to code and has been further improved in terms of conservatism reduction in papers such as in [14] where parameter-depende slack variables are considered or in [20] where slack variables are demonstrated to be related to sum-of-squares approach.

But one can easily notice that the LMI problem generated by this theorem is quite rapidly of huge dimensions. It may therefore be intractable in practice. The reasons for the numerical burden are on the one side the number of decision variables: one matrix $\Theta^{[i]}$ per vertex of the polytope. On the other side it is due to the size of the LMI (10) and to the size of the associated slack variable $H$. Ways to reduce this numerical burden in some special cases are explored in the remaining of the paper.

\subsection{Zero elements in the slack variable}

The slack variables can be seen as issued from application of Finsler lemma to inequality (5). Indeed, assume that the inequality holds for some parameter-dependent $\Theta(\xi)$. Finsler lemma then states the existence, for every uncertainty $\xi \in \Xi$, of a scalar $\tau(\xi)$ such that

$$
\Theta(\xi)>\tau(\xi)\left[\begin{array}{c}
\mathcal{E}_{1}^{*}(\xi) \\
-\mathcal{A}^{*}(\xi)
\end{array}\right]\left[\begin{array}{ll}
\mathcal{E}_{1}(\xi) & -\mathcal{A}(\xi)]
\end{array}\right.
$$


Take $\tau=\min _{\xi \in \Xi} \tau(\xi)$, the inequalities also hold for that minimal value. Introducing the matrix $H$ by addition and subtraction it gives

$$
\begin{aligned}
\Theta(\xi)> & \left(H\left[\mathcal{E}_{1}(\xi)-\mathcal{A}(\xi)\right]\right)^{\mathcal{H}} \\
& +\left(( \frac { \tau } { 2 } [ \begin{array} { c } 
{ \mathcal { E } _ { 1 } ^ { * } ( \xi ) } \\
{ - \mathcal { A } ^ { * } ( \xi ) }
\end{array} ] - H ) \left[\begin{array}{cc}
\mathcal{E}_{1}(\xi) & -\mathcal{A}(\xi)])^{\mathcal{H}}
\end{array}\right.\right.
\end{aligned}
$$

The slack variable approach of Theorem 4 amounts to the assumption of an affine polytopic $\Theta(\xi)$, i.e. $\Theta(\xi)=\sum_{i=1}^{\overline{1}} \xi_{i} \Theta^{[i]}$, and existence of a matrix $H$ such that the last term in (11) is sufficiently small for (10) to hold. "Good" choices of matrices $H$ are therefore such that this last term is close to zero, or negative semi-definite. Because of that, if the matrices $\mathcal{E}_{1}^{*}(\xi)$ and/or $\mathcal{A}^{*}(\xi)$ have zero rows for all uncertainties, then one can choose $H$ to have zero corresponding rows. Indeed, in such case these rows and columns will be zero in the last term of (11). Such choice is a recommended strategy to reduce the number of decision variables in $H$ when $\mathcal{E}_{1}(\xi)$ and/or $\mathcal{A}(\xi)$ have zero columns.

\subsection{Reduced slack variable}

This subsection is dedicated to a special case when the matrix $\mathcal{E}_{1}$ is not affected by uncertainties. This situation is the case for all usual LTI systems and for most descriptor systems. Recall that $\mathcal{E}_{1}$ is assumed invertible. In case it is not affected by uncertainties one can explicitly compute

$$
\left[\begin{array}{cc}
\mathcal{E}_{1} & -\mathcal{A}^{[i]}
\end{array}\right]^{\perp}=\left[\begin{array}{c}
\mathcal{E}_{1}^{-1} \mathcal{A}^{[i]} \\
\mathbf{1}
\end{array}\right]
$$

Define $a$ and $b$ as respectively the number of rows and columns of $\mathcal{A}$ and decompose $\mathcal{E}_{1}^{-1} \mathcal{A}^{[i]}$ according to the $a$ rows:

$$
\mathcal{E}_{1}^{-1} \mathcal{A}^{[i]}=\left[\begin{array}{c}
\mathcal{R}_{1}^{[i]} \\
\vdots \\
\mathcal{R}_{a}^{[i]}
\end{array}\right]
$$

Among these $a$ rows, assume $\underline{a} \leq a$ are uncertain. The indices of the uncertain rows are gathered in the set $\mathrm{L}\left(\mathrm{L}\right.$ contains $\underline{a}$ elements and if $k_{1} \notin \mathrm{L}$ then $\mathcal{R}_{k_{1}}^{[i]}=\mathcal{R}_{k_{1}}$ for all $\left.i=1 \ldots \overline{1}\right)$. Let the following algorithm:

\section{Algorithm 1}

0 - Initialize empty matrices $\mathcal{B}_{1}, \mathcal{B}_{2}, \mathcal{C}^{[i]}$ and integers $k_{1}=1, k_{2}=0$.

1 - If $k_{1} \in \mathrm{L}$ then increment $k_{2} \leftarrow k_{2}+1$, let the vector $e_{k_{2}}=\left(\begin{array}{lll}\mathbf{0}_{1,\left(k_{2}-1\right)} & 1 & \mathbf{0}_{1,\left(\underline{a}-k_{2}\right)}\end{array}\right)$ and append matrices according to

$$
\mathcal{B}_{1} \leftarrow\left[\begin{array}{c}
\mathcal{B}_{1} \\
e_{k_{2}}
\end{array}\right], \mathcal{B}_{2} \leftarrow\left[\begin{array}{c}
\mathcal{B}_{2} \\
\mathbf{0}_{1, b}
\end{array}\right], \mathcal{C}^{[i]} \leftarrow\left[\begin{array}{c}
\mathcal{C}^{[i]} \\
\mathcal{R}_{k_{1}}^{[i]}
\end{array}\right]
$$

2 - Else, $k_{1} \notin \mathrm{L}$, append matrices according to

$$
\mathcal{B}_{1} \leftarrow\left[\begin{array}{c}
\mathcal{B}_{1} \\
0_{1, \underline{a}}
\end{array}\right], \mathcal{B}_{2} \leftarrow\left[\begin{array}{c}
\mathcal{B}_{2} \\
\mathcal{R}_{k_{1}}
\end{array}\right]
$$

3 - Increment $k_{1} \leftarrow k_{1}+1$ and while $k_{1}<$ a go to step 1 .

Once this algorithm achieved, one gets for each vertex $i=1 \ldots \overline{1}$ the following factorization

$$
\left[\begin{array}{c}
\mathcal{E}_{1}^{-1} \mathcal{A}^{[i]} \\
\mathbf{1}_{b}
\end{array}\right]=\underbrace{\left[\begin{array}{cc}
\mathcal{B}_{1} & \mathcal{B}_{2} \\
\mathbf{0}_{b, \underline{a}} & \mathbf{1}_{b}
\end{array}\right]}_{\mathcal{D}}\left[\begin{array}{c}
\mathcal{C}^{[i]} \\
\mathbf{1}_{b}
\end{array}\right]
$$

where $\mathcal{B}_{2}$ gathers all the rows without uncertainties and $\mathcal{C}^{[i]}$ matrices have $\underline{a} \leq a$ rows. 
Theorem 5 If there exists a unique matrix $\hat{H}$ of appropriate dimensions and $\overline{1}$ IQS candidates $\Theta^{[i]}$ such that $\overline{\mathcal{L}}\left(\Theta^{[i]}\right)$ and

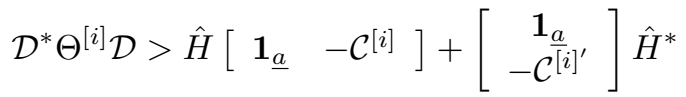

are feasible for all $i=1 \ldots \overline{1}$, then the interconnected loop (1) is well-posed whatever uncertainty $\xi \in \Xi$.

Compared to Theorem 4 this last result reduces the size of the main LMI constraint from $a+b$ to $\underline{a}+b$ rows and columns. Moreover, the number of decision variables in the slack variable $\hat{H}$ is reduced to $(\underline{a}+b) \underline{a}$ compared to the $(a+b) a$ elements of $H$. Reduction of the numerical burden is proportional to the square of the number of rows in $\mathcal{A}$ not affected by uncertainties $(a-\underline{a})$. Remains to demonstrate that the numerical complexity is reduced without conservatism augmentation.

Proposition 1 Assume $\mathcal{E}_{1}$ is not affected by uncertainties and let matrices $\mathcal{B}$ and $\mathcal{C}^{[i]}$ obtained following algorithm 1 then, if (10) holds, there exists some matrix $\hat{H}$ such that (13) holds as well.

ProofA: ccording to (12) one has

$$
\begin{aligned}
{\left[\begin{array}{ll}
\mathcal{E}_{1} & \left.-\mathcal{A}^{[i]}\right]
\end{array}\right] } & =\mathcal{E}_{1}\left[\begin{array}{ll}
\mathbf{1} & -\mathcal{B}_{1} \mathcal{C}^{[i]}-\mathcal{B}_{2}
\end{array}\right] \\
& =\mathcal{E}_{1}\left(\mathcal{B}_{1}\left[\mathcal{B}_{1}^{*}-\mathcal{C}^{[i]}\right]+\left[\begin{array}{ll}
\mathbf{1}-\mathcal{B}_{1} \mathcal{B}_{1}^{*} & -\mathcal{B}_{2}
\end{array}\right]\right) .
\end{aligned}
$$

Replace this value in inequality (10). Pre and post multiply the resulting inequality by $\mathcal{D}^{*}$ and $\mathcal{D}$ respectively. Notice that the matrices $\mathcal{B}_{1}, \mathcal{B}_{2}$ are such that $\mathcal{B}_{1}^{*} \mathcal{B}_{1}=\mathbf{1}_{\underline{a}}, \mathcal{B}_{1}^{*} \mathcal{B}_{2}=\mathbf{0}$, hence one obtains

$$
\mathcal{D}^{*} \Theta^{[i]} \mathcal{D}>\mathcal{D}^{*} H \mathcal{E}_{1} \mathcal{B}_{1}\left[\begin{array}{ll}
\mathbf{1}_{\underline{a}} & -\mathcal{C}^{[i]}
\end{array}\right]+\left[\begin{array}{c}
\mathbf{1}_{\underline{a}} \\
-\mathcal{C}^{[i]^{\prime}}
\end{array}\right] \mathcal{B}_{1}^{*} E_{1}^{*} \hat{H}^{*} \mathcal{D}
$$

which is exactly (13) with $\hat{H}=\mathcal{D}^{*} H \mathcal{E}_{1} \mathcal{B}_{1}$.

Note that for same reasons as in subsection 3.2 one may choose to force to zero some rows of $\hat{H}$ matrix if the corresponding columns of $\mathcal{C}^{[i]}$ are zero. It reduces the number of decision variables and is justified by Finsler lemma as exposed earlier.

The comparison of Theorems 4 and 5 is now done for the performance example considered earlier. For illustration purpose, assume $E=\mathbf{1}$ and assume both matrices $B(\xi)=B$ and $D(\xi)=D$ are not affected by uncertainties. Theorem 4 produces the following LMI constraints

$$
\begin{gathered}
{\left[\begin{array}{cccc|cc}
-P^{[i]} & \mathbf{0} & \mathbf{0} & \mathbf{0} & \mathbf{0} & \mathbf{0} \\
\mathbf{0} & \mathbf{0} & \mathbf{0} & \mathbf{0} & -P^{[i]} & \mathbf{0} \\
\mathbf{0} & \mathbf{0} & -\tau^{[i]} \mathbf{1} & \mathbf{0} & \mathbf{0} & \mathbf{0} \\
\mathbf{0} & \mathbf{0} & \mathbf{0} & -\tau^{[i]} \mathbf{1} & \mathbf{0} & \mathbf{0} \\
\hline \mathbf{0} & -P^{[i]} & \mathbf{0} & \mathbf{0} & \mathbf{0} & \mathbf{0} \\
\mathbf{0} & \mathbf{0} & \mathbf{0} & \mathbf{0} & \mathbf{0} & Q^{[i]}
\end{array}\right]} \\
\\
>
\end{gathered}
$$

to be tested for all vertices simultaneously. In comparison Theorem 5 produces the following LMI constraints

$$
\begin{aligned}
& {\left[\begin{array}{cc|cc}
\mathbf{0} & \mathbf{0} & -P^{[i]} & \mathbf{0} \\
\mathbf{0} & -\tau^{[i]} \mathbf{1} & \mathbf{0} & \mathbf{0} \\
\hline-P^{[i]} & \mathbf{0} & \mathbf{0} & \mathbf{0} \\
\mathbf{0} & \mathbf{0} & \mathbf{0} & -B^{*} P^{[i]} B-\tau^{[i]} D^{*} D+Q^{[i]}
\end{array}\right]} \\
& >\left(\hat{H}\left[\begin{array}{ll|ll}
\mathbf{1} & \mathbf{0} & -A^{[i]} & \mathbf{0} \\
\mathbf{0} & \mathbf{1} & -C^{[i]} & \mathbf{0}
\end{array}\right]\right)^{\mathcal{H}}
\end{aligned}
$$

which can be further reduced noticing that the last column of the $\mathcal{C}$ matrix is zero. Applying the strategy of subsection 3.2, it gives the set of reduced LMIs

$$
\begin{gathered}
{\left[\begin{array}{cc|c}
\mathbf{0} & \mathbf{0} & -P^{[i]} \\
\mathbf{0} & -\tau^{[i]} \mathbf{1} & \mathbf{0} \\
\hline-P^{[i]} & \mathbf{0} & \mathbf{0}
\end{array}\right]>\left(\tilde{H}\left[\begin{array}{cc|c}
\mathbf{1} & \mathbf{0} & -A^{[i]} \\
\mathbf{0} & \mathbf{1} & -C^{[i]}
\end{array}\right]\right)^{\mathcal{H}}} \\
Q^{[i]}>B^{*} P^{[i]} B+\tau^{[i]} D^{*} D
\end{gathered}
$$




\subsection{Unique separator}

An other, yet conservative, way to reduce numerical burden is to attempt solving constraints of Theorem 4 with a unique IQS for all uncertainties, i.e. $\Theta^{[i]}=\Theta$ for all $i=1 \ldots \overline{1}$. This corresponds in classical robustness theory literature to the "quadratic stability" concept, see [2], where a unique Lyapunov matrix is used for all realization of an uncertain system. But in classical "quadratic stability" framework there is no need of slack variables and the LMIs are of reduced size. This situation corresponds to the following theorem.

Theorem 6 Assume $\mathcal{E}_{1}$ is not affected by uncertainties, then if there exists a unique IQS candidate $\Theta$ such that $\overline{\mathcal{L}}(\Theta)$ and

$$
\left[\begin{array}{c}
\mathcal{E}_{1}^{-1} \mathcal{A}^{[i]} \\
\mathbf{1}
\end{array}\right]^{*} \Theta\left[\begin{array}{c}
\mathcal{E}_{1}^{-1} \mathcal{A}^{[i]} \\
\mathbf{1}
\end{array}\right]>\mathbf{0}
$$

are feasible for all $i=1 \ldots \overline{\mathbf{1}}$ with the additional constraint that $\left[\begin{array}{ll}\mathbf{1}_{a} & \mathbf{0}\end{array}\right] \Theta\left[\begin{array}{ll}\mathbf{1}_{a} & \mathbf{0}\end{array}\right]^{*} \leq \mathbf{0}$, then the interconnected loop (1) is well-posed whatever uncertainty $\xi \in \Xi$.

The theorem holds because of the convexity of the constraints with respect to the uncertain data $\mathcal{A}^{[i]}$ as soon as the upper-left block of the IQS $\Theta$ matrix is negative semi-definite. Moreover, recall (see section 2.3) that the constraint on the upper left block of $\Theta$ to be negative semi-definite is not restrictive in most cases.

Numerically, LMIs of Theorem 6 are much less demanding than those of Theorem 4, but the results are proved to be always more conservative as attested by the following proposition.

Proposition 2 Assume there exists a solution $\Theta$ to the constraints of Theorem 6 , then there exists a unique $I Q S \Theta^{[i]}=\Theta$ and a matrix $H$ solution to constraints of Theorem 4 .

Proof: The proof is done assuming that the upper-left block of $\Theta$ is negative definite, but by a small perturbation argument it holds as well if it has some zero eigenvalues. Decompose $\Theta$ in blocks as follows $\Theta=\left[\begin{array}{ll}\Theta_{11} & \Theta_{12} \\ \Theta_{12}^{*} & \Theta_{22}\end{array}\right]$ where $\Theta_{11}=\left[\begin{array}{ll}\mathbf{1}_{a} & \mathbf{0}\end{array}\right] \Theta\left[\begin{array}{ll}\mathbf{1}_{a} & \mathbf{0}\end{array}\right]^{*}$. By Schur complement argument, inequality (14) implies that

$$
\left[\begin{array}{cc}
-\Theta_{11} & \Theta_{11} \mathcal{E}_{1}^{-1} \mathcal{A}^{[i]} \\
\left(\mathcal{E}_{1}^{-1} \mathcal{A}^{[i]}\right)^{*} \Theta_{11} & \left(\Theta_{12}^{*}\left(\mathcal{E}_{1}^{-1} \mathcal{A}^{[i]}\right)\right)^{\mathcal{H}}+\Theta_{22}
\end{array}\right]>\mathbf{0}
$$

It also reads as

$$
\Theta>\left[\begin{array}{cc}
2 \Theta_{11} & \Theta_{12}-\Theta_{11} \mathcal{E}_{1}^{-1} \mathcal{A}^{[i]} \\
\Theta_{12}^{*}-\left(\mathcal{E}_{1}^{-1} \mathcal{A}^{[i]}\right)^{*} \Theta_{11} & -\left(\Theta_{12}^{*}\left(\mathcal{E}_{1}^{-1} \mathcal{A}^{[i]}\right)\right)
\end{array}\right]
$$

which is nothing but equation (10) when taking

$$
H=\left[\begin{array}{c}
\Theta_{11} \\
\Theta_{12}^{*}
\end{array}\right] \mathcal{E}_{1}^{-1}
$$

Notice that Proposition 2 not only states that "quadratic stability" type results are always more conservative than the general slack variable result, but it also states that if one looks for a parameter-independent separator $\Theta=\Theta^{[i]}$, then slack variables are useless. With the restriction $\Theta=\Theta^{[i]}$, the numerical complexity of Theorem 6 is reduced without conservatism compared to Theorem 4.

\section{Conclusion}

The study related to numerical issues of the slack variable technique that is exposed in the paper has been motivated by questions raised while coding the RoMulOC toolbox, [15]. At this stage, the toolbox includes results that may be understood as corresponding to Theorems 4 and 6 . When tested on numerical examples (for example those of the project described in [1]), the latter theorem produces quite conservative results while the former too frequently fails due to numerical problems. Not only the numerical burden of large LMI problems makes the optimization long, but it seems that the unnecessary decision variables (as attested by Proposition 1) have an effect on the accuracy of the solvers. A next version of the RoMulOC toolbox is currently being coded and will include results of Theorem 5 . 


\section{References}

[1] D. Arzelier and D. Peaucelle. Multiobjective $H_{2} / H_{\infty} /$ impulse-to-peak synthesis: Application to the control of an aerospace launcher. In IFAC Symposium on Automatic Control in Aerospace, St. Petersburg, Russia, June 2004.

[2] B.R. Barmish. Necessary and sufficient conditiond for quadratic stabilizability of an uncertain system. J. Optimization Theory and Applications, 46(4), August 1985.

[3] M.C. de Oliveira, J. Bernussou, and J.C. Geromel. A new discrete-time stability condition. Systems \& Control Letters, 37(4):261-265, July 1999.

[4] M.C. De Oliveira and R.E. Skelton. Perspectives in Robust Control, chapter Stability tests for constrained linear systems, pages 241-257. Lecture Notes in Control and Information Sciences. Springer, 2001. edited by S.O. Reza Moheimani.

[5] Y. Ebihara and T. Hagiwara. New dilated LMI characterizations for continuous-time multiobjective controller synthesis. Automatica, 40(11):2003-2009, 2004.

[6] L. El Ghaoui and S.-I. Niculescu, editors. Advances in Linear Matrix Inequality Methods in Control. Advances in Design and Control. SIAM, Philadelphia, 2000.

[7] F. Gouaisbaut and D. Peaucelle. A note on stability of time delay systems. In IFAC Symposium on Robust Control Design, Toulouse, July 2006.

[8] F. Gouaisbaut and D. Peaucelle. Robust stability of time-delay systems with interval delays. In IEEE Conference on Decision and Control, New Orleans, December 2007.

[9] T. Iwasaki and S. Hara. Well-posedness of feedback systems: Insights into exact robustness analysis and approximate computations. IEEE Trans. on Automat. Control, 43(5):619-630, 1998.

[10] T. Iwasaki and S. Hara. Generalized KYP lemma: Unified frequency domain inequalities with design applications. IEEE Trans. on Automat. Control, 50(1):41-59, January 2005.

[11] T. Iwasaki and G. Shibata. LPV system analysis via quadratic separator for uncertain implicit systems. IEEE Trans. on Automat. Control, 46(8):1195-1207, August 2001.

[12] U Jönsson. Lectures on input-output stability and integral quadratic constraints. http://www.math.kth.se/ ulfj/5B5744/Lecturenotes.ps, 2001.

[13] A. Megreski and A. Rantzer. System analysis via integral quadratic constraints. IEEE Trans. on Automat. Control, 42(6):819-830, June 1997.

[14] R.C.L.F. Oliveira, M.C. de Oliveira, and P.L.D. Peres. Convergent LMI relaxations for robust analysis of uncertain linear systems using lifted polynomial parameter-dependent Lyapunov functions. Systems \& Control Letters, 57:680-689, 2008.

[15] D. Peaucelle. RoMulOC: a YALMIP-Matlab based robust multi-objective control toolbox. Technical Report 05377, LAAS-CNRS, Toulouse, June 2005.

[16] D. Peaucelle. Quadratic separation for uncertain descriptor system analysis, strict LMI conditions. In IEEE Conference on Decision and Control, New Orleans, December 2007. Section 5 of the manuscript is erroneous, a corrected version is available at www.laas.fr/ peaucell/papers/cdc07a.pdf.

[17] D. Peaucelle, D. Arzelier, O. Bachelier, and J. Bernussou. A new robust D-stability condition for real convex polytopic uncertainty. Systems \& Control Letters, 40(1):21-30, May 2000.

[18] D. Peaucelle, D. Arzelier, D. Henrion, and F. Gouaisbaut. Quadratic separation for feedback connection of an uncertain matrix and an implicit linear transformation. Automatica, 43:795-804, 2007. doi: 10.1016/j.automatica.2006.11.005.

[19] D. Peaucelle, L. Baudouin, and F. Gouaisbaut. Integral quadratic separators for performance analysis. In European Control Conference, Budapest, August 2009. Submitted, a technical note version is available at ww. laas.fr/ peaucell/papers/ecc09.pdf. 
[20] D. Peaucelle and M. Sato. LMI tests for positive definite polynomials: Slack variable approach. IEEE Trans. on Automat. Control, 2009. To appear.

[21] M.G. Safonov. Stability and Robustness of Multivariable Feedback Systems. Signal Processing, Optimization, and Control. MIT Press, 1980.

[22] C.W. Scherer. Advances in Linear Matrix Inequality Methods in Control, chapter 10 Robust Mixed Control and Linear Parameter-Varying Control with Full Block Scallings, pages 187-207. Advances in Design and Control. SIAM, 2000. edited by L. El Ghaoui and S.-I. Niculescu.

[23] C.W. Scherer. Relatations for robust linear matrix inequality problems with verifications for exactness. SIAM J. Matrix Anal. Appl., 27(2):365-395, 2005.

[24] A. Trofino Neto. Parameter dependent Lyapunov functions for a class of uncertain linear systems: an LMI approach. In IEEE Conference on Decision and Control, pages 2341-2346, Phoenix, December 1999.

[25] J. Zhang, C.R. Knospe, and Tsiotras. New results for the analysis of linear systems with time invariant delays. International Journal of Robust and Nonlinear Control, 13:1149-1175, 2003. 